Claremont Colleges

Scholarship@ Claremont

Pomona Faculty Publications and Research

Pomona Faculty Scholarship

$1-30-2002$

\title{
Observation of Anomalous Spin-State Segregation in a Trapped Ultracold Vapor
}

Dwight L. Whitaker

Pomona College

H. J. Lewandowski

D. M. Harber

E. A. Cornell

\section{Recommended Citation}

"Observation of Anomalous Spin-State Segregation in a Trapped Ultracold Vapor," H. J. Lewandowski, D. M. Harber, D. L. Whitaker, and E. A. Cornell, Phys. Rev. Lett., 88, 070403, (2002). DOI: 10.1103/PhysRevLett.88.070403.

This Article is brought to you for free and open access by the Pomona Faculty Scholarship at Scholarship @ Claremont. It has been accepted for inclusion in Pomona Faculty Publications and Research by an authorized administrator of Scholarship @ Claremont. For more information, please contact scholarship@cuc.claremont.edu. 


\title{
Observation of Anomalous Spin-State Segregation in a Trapped Ultracold Vapor
}

\author{
H. J. Lewandowski, D. M. Harber, D. L. Whitaker,* and E. A. Cornell ${ }^{\dagger}$ \\ JILA, National Institute of Standards and Technology and Department of Physics, University of Colorado, \\ Boulder, Colorado 80309-0440
}

(Received 25 September 2001; published 30 January 2002)

\begin{abstract}
We observe counterintuitive spin segregation in an inhomogeneous sample of ultracold, noncondensed rubidium atoms in a magnetic trap. We use spatially selective microwave spectroscopy to verify a model that accounts for the differential forces on two internal spin states. In any simple understanding of the cloud dynamics, the forces are far too small to account for the dramatic transient spin polarizations observed. The underlying mechanism remains to be elucidated.
\end{abstract}

DOI: $10.1103 /$ PhysRevLett.88.070403

Imagine a near-ideal gas consisting of a mixture of two distinct molecular species. Apply a differential force that tends to pull the two species apart. What determines whether the two species segregate or remain mixed? From a kinetics point of view, the question becomes whether the segregating drift velocity, a function of the differential force and the mean free path, is larger or smaller than the effective velocity of remixing diffusion. The "diffusive velocity" depends on the characteristic size of the sample. The equilibrium point of view focuses instead on whether the energetic benefit derived from separating in the differential potential outweighs the corresponding entropic cost. Again, for a given magnitude force, the ultimate outcome hinges on the size of the sample. Both points of view lead to the same quantitative criterion - is the differential potential across the sample large or small compared to the thermal energy $k_{B} T$ ? As an example, a mixture of gaseous oxygen and nitrogen in a room-sized box does not undergo separation under the differential force of Earth's gravity, because the gravitational potential of the differential mass ( $4 \mathrm{amu}$ ), evaluated over a few meters, is very small compared to $k_{B} T$.

In this paper, we study the segregation behavior of a gaseous mixture. Our system consists of magnetically trapped ultracold rubidium gas in two spin states. These states can be interconverted in the presence of an applied microwave magnetic field, but in the absence of the applied drive the two states do not interconvert and can be thought of as two distinct fluids [1]. Interatomic interactions and differential magnetic moments give rise to a small differential potential that would tend to separate the two species, except that it is a thousand times smaller than $k_{B} T$. In contrast to the above arguments we observe that the initially homogenized sample nonetheless undergoes a transient segregation.

In the first part of this paper, we describe the use of spatially selective Ramsey spectroscopy to characterize a differential potential acting on the two spin states. In the second part, we describe our observations of the atomic motion induced by the differential potential over longer time scales. The atomic motions are much too large to be
PACS numbers: 05.30.Jp, 32.80.Pj, 51.60. +a, 75.30.Ds

described simply as arising from an acceleration from the differential potential.

The experimental apparatus includes a single magnetooptical vapor-cell trap which precools a sample of ${ }^{87} \mathrm{Rb}$ atoms for transfer into a quadrupole magnetic trap. The electromagnetic quadrupole trap coils are mounted on a servo-controlled linear track that mechanically moves the atoms to a UHV region where they are transferred into a Ioffe-Pritchard magnetic trap for further cooling via rf evaporation. Our Ioffe-Pritchard trap uses permanent magnets to produce strong radial confinement. Electromagnetic coils generate a stable and adjustable bias field and a weak confining potential in the axial direction. The trapping frequencies are $\{7,230,230\} \mathrm{Hz}$. Typical sample sizes are on the order of $10^{6}$ atoms at a temperature of $850 \mathrm{nK}$ (several times the Bose-Einstein condensation temperature).

The two hyperfine states of interest are $|1\rangle \equiv \mid F=$ $\left.1, m_{f}=-1\right\rangle$ and $|2\rangle \equiv\left|F=2, m_{f}=1\right\rangle$. We coherently couple the two hyperfine states via a two-photon drive [2]. The drive consists of a microwave photon $(\sim 6.8 \mathrm{GHz})$ and a rf photon $(1-3 \mathrm{MHz})$. The sum frequency is detuned $200 \mathrm{~Hz}$ from the transition, and the effective two-photon Rabi frequency is $2.5 \mathrm{kHz}$. The drive is turned on for $\sim 100 \mu$ s to transfer half of the atoms (initially all in the $|1\rangle$ state) to the $|2\rangle$ state (a $\frac{\pi}{2}$ pulse). A second $\frac{\pi}{2}$ pulse is applied after a variable delay. The final population and spatial distribution in either spin state can be separately determined using absorption imaging.

We determine the transition frequency between states $|1\rangle$ and $|2\rangle$ by varying, in each realization of the experiment, the delay time between the two $\frac{\pi}{2}$ pulses and measuring the relative population in the $|1\rangle$ state. Typical delay times range from 1-60 ms. The frequency of the resulting Ramsey fringes is the difference between the atomic transition and the oscillator frequency of our two-photon drive [3]. In order to study the spatial variation of the transition frequency between the two states, we break up the images into nine bins along the axial direction and integrate the number of atoms in each bin (Fig. 1).

There are two different mechanisms that shift the transition frequency. The first, dominant in the limit of low 
(a)

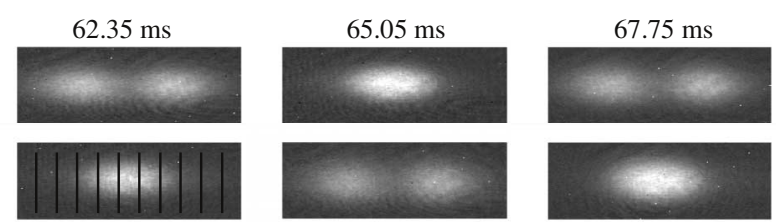

(b)

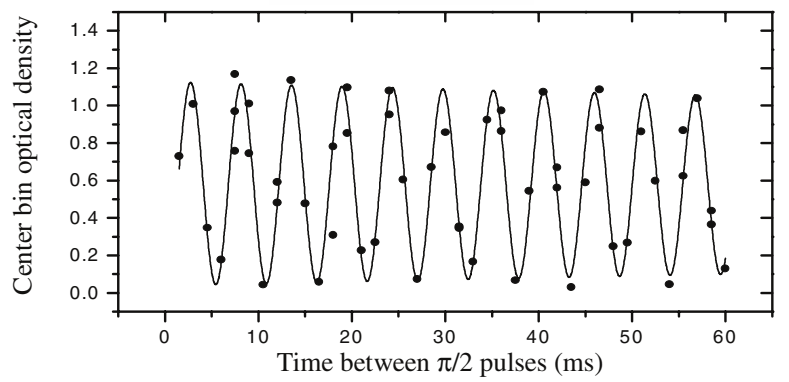

(c)

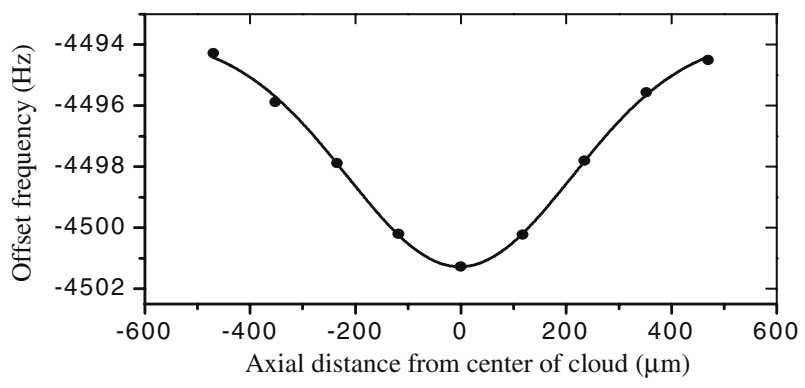

FIG. 1. (a) Images of $F=1$ atoms (upper) and $F=2$ atoms (lower) after two $\frac{\pi}{2}$ pulses. The delay between the pulses is listed at the top. The edges of the cloud have become out of phase with the center. The black lines show how the cloud is divided up along the axial direction to measure the spatial dependence of the transition frequency. Each image is $1 \mathrm{~mm}$ on the horizontal (axial) dimension. (b) The center-bin $|1\rangle$ optical density as a function of time between the two $\frac{\pi}{2}$ pulses. (c) The plot shows the spatial dependence of the transition frequency in the axial direction. Offset frequency is defined as the transition frequency $-6834682610.9 \mathrm{~Hz}$. The solid line is a Gaussian fit to the data. These data were taken at $B_{\text {bias }}=3.23 \mathrm{G}$.

atomic density, is the differential Zeeman effect. The atoms are confined in the parabolic minimum of the magnetic field and each spin state individually experiences a Zeeman shift, which varies by $30 \mathrm{kHz}$ from the center to the edge of the cloud [4]. States $|1\rangle$ and $|2\rangle$ were selected because the Zeeman shifts cancel to great accuracy. The Breit-Rabi equation [5] predicts that the frequency splitting is first-order independent of field at $3.23 \mathrm{G}$, increasing quadratically with magnetic field about that minimum. The magnetic field in turn has a minimum value, $B_{\text {bias }}$, at the center of the magnetic trap, and increases quadratically with displacement along the trap axis. Thus for a $B_{\text {bias }}=$ $3.23 \mathrm{G}$, the differential Zeeman shift across the cloud is quartic in axial position; the quartic shift from trap center to sample edge is $0.7 \mathrm{~Hz}$. For $\left|B_{\text {bias }}-3.23 \mathrm{G}\right|>0.02 \mathrm{G}$, the dominant field dependence with position is quadratic. For instance, for $B_{\text {bias }}=3 \mathrm{G}$, the frequency shift from cloud center to edge is $-7 \mathrm{~Hz}$.

The second frequency shift is due to the mean-field energy that arises from the self-interaction of the atoms. The two states have slightly different $s$-wave scattering lengths and therefore different interaction energies. The shift of the transition energy is given by

$$
\Delta E_{m f}=\frac{4 \pi \hbar^{2}}{m}\left[2 n_{2} a_{22}-2 n_{1} a_{11}+2\left(n_{1}-n_{2}\right) a_{12}\right]
$$

where $a_{i j}$ is the $s$-wave scattering length between states $|i\rangle$ and $|j\rangle, n_{i}$ is the density in state $|i\rangle$, and the prefactors of 2 account for the enhancement of $s$-wave collisions for noncondensed bosons. For ${ }^{87} \mathrm{Rb}$ the $s$-wave scattering lengths are $a_{11}=100.9 a_{0}, a_{22}=95.6 a_{0}$, and $a_{12}=$ $98.2 a_{0}$ [6] where $a_{0}$ is the Bohr radius. The mean-field shift has very little dependence on relative populations in the two states because $a_{12} \simeq\left(a_{11}+a_{22}\right) / 2$. After a $\frac{\pi}{2}$ pulse, $n_{1} \approx n_{2} \approx n / 2$, and to sufficient accuracy $\Delta E_{m f}=\left(a_{22}-a_{11}\right) 4 \pi \hbar n / m$.

We measure the mean-field shift spectroscopically. The sample density is inhomogeneous, with a Gaussian profile in each dimension. The radial oscillation period is short compared to the duration of the Ramsey measurement and to the mean collision time $(\sim 10 \mathrm{~ms})$. Thus, the random thermal motion of the atoms effectively averages over the radial density inhomogeneity. The axial oscillation period, on the other hand, is comparable to the measurement duration and long compared to the collision time. To a reasonable approximation one can treat the atoms as "frozen" in the axial direction. To further reduce the effect of axial density inhomogeneity, we use the data only from the center bin, a region of relative uniformity. We create clouds with various peak densities and measure the transition frequency of the center bin. The slope of the frequency vs density fit $\left(-0.52 \times 10^{12} \mathrm{~Hz} / \mathrm{cm}^{3}\right)$ is more negative than a prediction based on photoassociative data by $20 \%$ [6]. However, it is entirely possible that our analysis of our cloud images could have systematically underestimated the cloud density by as much as $20 \%$. More troubling is that the zero-density extrapolation, at $6834678118.0(7) \mathrm{Hz}$ at $3.23 \mathrm{G}$, is too high by 4.4(7) Hz. We are unable to account for this systematic offset.

For a particular peak density we measure the axial spatial variation in frequency by binning the cloud into several sections and determining a frequency for each bin. This measurement is done at $B_{\text {bias }}=3.23 \mathrm{G}$ to minimize the magnetic contribution to the inhomogeneity. The transition frequency vs axial position along the cloud is shown in Fig. 1c.

We can change the frequency shift across the cloud and nearly cancel both of the frequency shifts by operating at a different value of $B_{\text {bias }}$. The cancellation of the mean-field and magnetic frequency shifts cannot be perfect because the cloud has a Gaussian density profile and the magnetic field shift is essentially parabolic across the cloud. However, for a cloud with $2 \times 10^{6}$ atoms at a temperature of $500 \mathrm{nK}$ there will be less than $1 \mathrm{~Hz}$ shift across the cloud at $B_{\text {bias }}=2.83 \mathrm{G}$ (Fig. 2). 


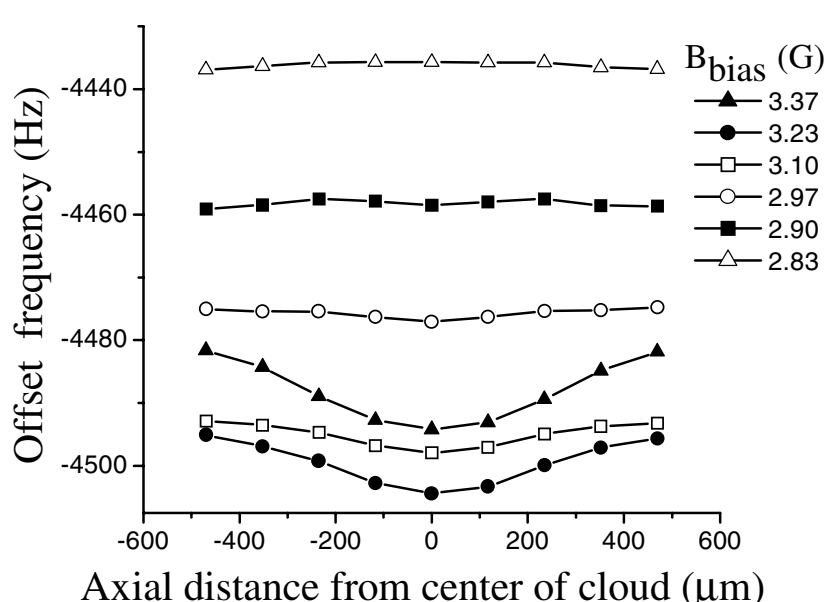

Axial distance from center of cloud $(\mu \mathrm{m})$

FIG. 2. The transition frequencies at different positions along the cloud are plotted as a function of $B_{\text {bias. }}$. The cloud has a peak density of $4 \times 10^{13} \mathrm{~cm}^{-3}$. Offset frequency is defined as the transition frequency $-6834682610.9 \mathrm{~Hz}$. The frequency shift at $3.23 \mathrm{G}$ (negligible magnetic contribution to the inhomogeneity) has a Gaussian shape. At $B_{\text {bias }}=3.37 \mathrm{G}$ the magnetic shift and the mean-field shift are in the same direction, and therefore the inhomogeneity across the cloud is increased. When the cloud is in a field of $2.83 \mathrm{G}$, inhomogeneity due to the mean-field shift is almost completely canceled by the magnetic shift. Data points are connected to guide the eye.

Conversely, if we operate at $B_{\text {bias }}>3.23 \mathrm{G}$, the curvature of the magnetic contribution to the frequency inhomogeneity adds with the same sign to that from the density contribution, and we see enhanced total frequency shift across the cloud (Fig. 2). Near the center of the cloud, the frequency shift is parabolic with displacement from the center, and the curvature of the differential potential can be conveniently characterized by

$$
\left.\nu_{\text {diff }}=1 / 2 \pi \sqrt{h / m \times\left\langle d^{2} \nu_{12} / d z^{2}\right.}\right\rangle,
$$

where $m$ is the mass of ${ }^{87} \mathrm{Rb}$ and $\nu_{12}$ is the transition frequency [7]. $\nu_{\text {diff }}$ may be thought of as the axial motional frequency of a hypothetical test atom, in state $|2\rangle$, under the influence of only the differential magnetic and mean-field forces. For the data shown in Fig. 2, measured $\nu_{\text {diff }}$ ranges from $0.15 \mathrm{~Hz}$ for $B_{\text {bias }}=3.37 \mathrm{G}$, down through $0.1 i \mathrm{~Hz}$ for $B_{\text {bias }}=2.83 \mathrm{G}$.

We have used spectroscopic methods to characterize the spatial dependence of the differential potential experienced by states $|1\rangle$ and $|2\rangle$ in our trap. The data are quantitatively accounted for by a simple model involving magnetic and mean-field potentials. We now describe the observed mechanical effects that the gradients of these potentials have on the distribution of the relative atomic densities of states $|1\rangle$ and $|2\rangle$.

In the twin-pulse spectroscopy described so far, the first pulse sets up a coherent superposition of the two states; the delay time between the two pulses allows the relative internal phase, $\phi$, in the coherent superposition $\left(|1\rangle+e^{i \phi}|2\rangle\right)$ to evolve under the inhomogeneous potential, and the second pulse then converts the spatial pattern in $\phi$ into a pattern of relative density for ready imaging. In the experiments described below, the second $\frac{\pi}{2}$ pulse is omitted, and the delay between the first pulse and the density imaging is increased to allow time for the atoms to move under the influence of the developing spatial gradient in $\phi$. The patterns in the density distribution which we image must result from the physical displacement of atoms during the period after the $\frac{\pi}{2}$ pulse [8].

The results of this class of experiment are summarized in Fig. 3. Starting around $50 \mathrm{~ms}$ after the $\frac{\pi}{2}$ pulse, the atoms in the $|1\rangle$ state are observed to move outward along the trap axis, while the atoms in the $|2\rangle$ state move inward. Maximum separation is realized at about $100 \mathrm{~ms}$, then the two clouds relax back to their original, well-overlapped distribution by around $200 \mathrm{~ms}$. No further evolution is

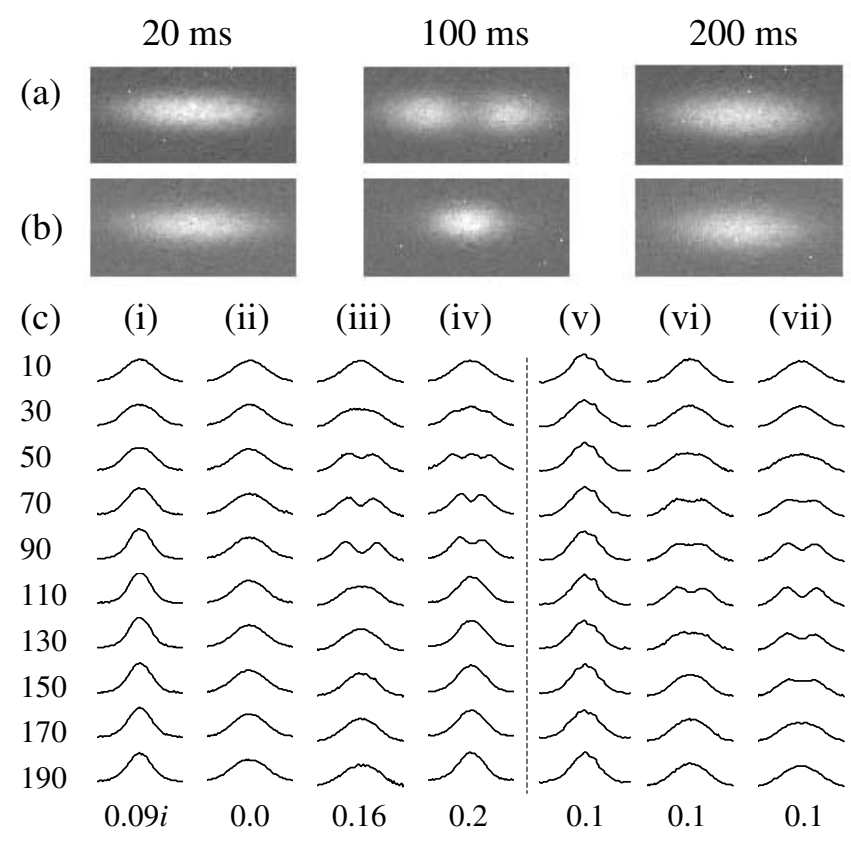

FIG. 3. (a) Spin segregation appears in the spatial distribution of atoms in the $|1\rangle$ state at various times after the $\frac{\pi}{2}$ pulse. |1) state atoms move outward, then relax back to their initial condition. Initial cloud conditions are peak total density $1.8 \times 10^{13} \mathrm{~cm}^{-3}, T=850 \mathrm{nK}, \nu_{\text {diff }}=0.1 \mathrm{~Hz}$. (b) Same as for (a), but showing the $|2\rangle$ state distribution. $|1\rangle$ and $|2\rangle$ state motions are complementary, and leave the total density profile unchanged. (c) $|1\rangle$-state axial density profile imaged at times after the $\frac{\pi}{2}$ pulse, listed on the left column. The cross sections have been normalized to the data at $10 \mathrm{~ms}$ for each column. For all columns, $T=850 \mathrm{nK}$. $\nu_{\text {diff }}$ values are listed at the bottom of each column. The first column of cross sections has an imaginary $\nu_{\text {diff }}$ corresponding to negative curvature of the frequency. Initial density (in $10^{13} \mathrm{~cm}^{-3}$ ) and $B_{\text {bias }}$ (in gauss) are for each column; (i) 1.8, 2.99; (ii) 1.8, 3.12; (iii) 1.8, 3.52; (iv) 1.8, 3.79; (v) $0.25,3.35$; (vi) $1.2,3.31$; (vii) $2.0,3.27$. Columns (i)-(iv) are at fixed density and progressively higher $\nu_{\text {diff }}$, showing turn-on of effect with increasing $\nu_{\text {diff }}$. Columns (v)-(vii) are at fixed $\nu_{\text {diff }}$ and at progressively higher density, showing turn-on of segregation effect with density. 
observed. The spin segregation is dependent on the curvature of the differential potential. For $\left|\nu_{\text {diff }}\right|<0.05 \mathrm{~Hz}$ [Fig. 3(ii)], no separation is observed above the imaging noise. For $\nu_{\text {diff }}>0.18 \mathrm{~Hz}$ [Fig. 3(iv)], we observe what we call "higher-order" effects, with the relative spindensity developing multiple stripes.

We find the segregation to be startling. While the meanfield and magnetic forces can combine to provide a small buoyant force that tends to push the $|1\rangle$ atoms out towards the lower density regions of the clouds, the magnitude of the observed effect is entirely disproportionate. For $\nu_{\text {diff }}=0.1 \mathrm{~Hz}$, for instance, the total frequency shift $\Delta \nu_{12}$ from center to edge of cloud is $6 \mathrm{~Hz}$. This should be compared to $k_{B} T / h \sim 10 \mathrm{kHz}$. The criterion for separation discussed in the introduction is thus clearly not satisfied. This objection would be surmounted if the initially well-homogenized and noncondensed populations in each spin state nonetheless moved collectively [the relevant inequality would then be $N_{\text {atoms }}\left(\Delta \nu_{12} h\right) \gg k T$ rather than $\Delta \nu_{12} h \ll k T$ ] [9]. One would still need to account, however, for the rate of segregation. If the atoms move ballistically in the differential potential, the minimum time for the peak segregation to develop should be at least $1 / 4 \nu_{\text {diff }}$ [9]. But the observed segregation times are at least 10 times faster.

As seen in Fig. 3c, to induce segregation one needs not only a sufficiently large value of $\nu_{\text {diff }}$ but independently one needs adequate density. It is possibly significant that the segregation seems to disappear when the density gets so low that the cloud is no longer collisionally thick in the axial direction. While the observed segregation rate is fast compared to $4 \nu_{\text {diff }}$, it is slow compared to $4 \nu_{z}$, where $\nu_{z}$ is the axial trap frequency. That is, the random thermal speed of the atoms, if it were selectively directed, is more than adequate to move the atoms the required distance. We speculate that as the internal phase $\phi$ develops a spatial gradient, it somehow acts as a "switch" to control velocity-changing collisions such that atoms in the $|2\rangle$ state are preferentially scattered inward and $|1\rangle$ atoms outward.

Finally, the observation of nondiffusive evolution of spin populations compels a comparison with the spin-wave phenomenon observed [10] and discussed extensively [11] in the 1980s. We understand the earlier work to be an effect of the transverse spin. The present work describes evolution of the relative population of energy eigenstates, a longitudinal spin effect. Additional study may lead to connections between the two spin effects.

We acknowledge useful conversations with the other members of the JILA BEC Collaboration and with Kurt Gibble. This work is supported by the NSF and by NIST.
*Present address: Physics Department, Williams College, Williamstown, MA 01267.

${ }^{\dagger}$ Quantum Physics Division, National Institute of Standards and Technology.

[1] E. Cornell, D. Hall, M. Matthews, and C. Wieman, J. Low Temp. Phys. 113, 151 (1998).

[2] M. R. Matthews et al., Phys. Rev. Lett. 81, 243 (1998).

[3] N. F. Ramsey, Molecular Beams (Clarendon Press, Oxford, 1956).

[4] We arbitrarily define the "edges" of the cloud to correspond to $\pm 1.5 \times$ HWHM.

[5] From the Breit-Rabi equation we expand the relative Zeeman shift about the minimum: $\nu=6834678113.59(2) \mathrm{Hz}+431.35957(9) \mathrm{Hz} / \mathrm{G}^{2} \times$ $[B-3.228917(3) \mathrm{G}]^{2}$. These values and associated uncertainties are derived from the zero-field hyperfine splitting $6834682610.90434(3)$ [12] and the nuclear $g$ factor $0.0009951414(10)$ [13].

[6] J. M. Vogels, R. S. Freeland, C. C. Tsai, B. J. Verhaar, and D. J. Heinzen, Phys. Rev. A 61, 043407 (2000); D. J. Heinzen (private communication).

[7] We use a density weighted frequency curvature defined by $\left\langle\frac{d^{2} \nu_{12}}{d z^{2}}\right\rangle=\int_{-\infty}^{\infty} n(z) \frac{d^{2} \nu_{12}}{d z^{2}}(z) d z / \int_{-\infty}^{\infty} n(z) d z$ where $n(z)$ is the axial Gaussian density profile.

[8] In the absence of applied microwave, spin population does not spontaneously interconvert. The energy released by a single atom converting from state $|2\rangle$ to state $|1\rangle$, if it were thermalized among the sample of $10^{6}$ atoms, would result in a $50 \%$ increase in sample temperature. This warming is not observed. The spin populations must thus each independently obey a mass continuity equation.

[9] Indeed, exactly such behavior was observed in the collective motion of a two-level Bose-Einstein condensate [14]. The observed condensate segregation rate was in good agreement with the corresponding value of $\nu_{\text {diff }}$. That is, the condensates' segregation was at least 10 times slower than the present noncondensate effect.

[10] B. R. Johnson et al., Phys. Rev. Lett. 52, 1508 (1984); N. P. Bigelow, J. H. Freed, and D. M. Lee, Phys. Rev. Lett. 63, 1609 (1989); W. J. Gully and W. J. Mullin, Phys. Rev. Lett. 52, 1810 (1984).

[11] C. Lhuillier and F. Laloë, J. Phys. (Paris) 43, 225 (1982); 43, 197 (1982); A. Leggett and M. Rice, Phys. Rev. Lett. 20, 586 (1968); 21, 506(E) (1968); K. Miyake, W. Mullin, and P. C. E. Stamp, J. Phys. (Paris) 46, 663 (1985); L. P. Lévy and A. E. Ruckenstein, Phys. Rev. Lett. 52, 1512 (1984); G. Tastevin, P. J. Nacher, M. Luduc, and F. Laloë, J. Phys. Lett. 46, 249 (1985).

[12] C. Salomon et al., in Proceedings of the 17th International Conference on Atomic Physics (ICAP 2000), edited by E. Arimondo, P.D. Natale, and M. Inguscio (American Institute of Physics, New York, 2001), p. 23.

[13] E. Arimondo, M. Inguscio, and P. Violino, Rev. Mod. Phys. 49, 31 (1977).

[14] D. Hall et al., Phys. Rev. Lett. 81, 1539 (1998). 